\title{
Build and Optimization of an Intelligent Decision Support System for UGVs Based on the Human-Robot Coordination
}

\author{
Li Jing ${ }^{1}$, Tang Zhenmin ${ }^{2}$, Tan Yefa ${ }^{1}$, Shi Zhaoxia ${ }^{2}$, Liu Jiayin ${ }^{2}$ \\ ${ }^{1}$ College of Field Engineering, PLA University of Science \& Technology, Nanjing Jiangsu, China \\ ${ }^{2}$ Department of Computer Science and Engineering, Nanjing University of Science and Technology, Nanjing Jiangsu, China
}

\section{Email address:}

lijingwry@126.com (Li Jing), tang.zm@163.com (Tang Zhenmin), tanyefa7651@162.com (Tan Yefa), stonexia@soho.com (Shi Zhaoxia), smiton@foxmail.com (Liu Jiayin)

\section{To cite this article:}

Li Jing, Tang Zhenmin, Tan Yefa, Shi Zhaoxia, Liu Jiayin. Build and Optimization of an Intelligent Decision Support System for UGVs Based on the Man-Robot Coordination. Science Discovery. Vol. 4, No. 1, 2016, pp. 6-11. doi: 10.11648/j.sd.20160401.12

Received: January 29, 2016; Accepted: February 18, 2016; Published: April 8, 2016

\begin{abstract}
Unmanned systems of autonomy is a kind of perception, observation, analysis, communication, planning, decision-making and action ability, and complete human tasks assigned by the human-computer interaction it. Ground unmanned systems or in academic research field known as mobile robot autonomous land vehicle, known as the unmanned ground vehicles in the army. Based on variable independently, fault diagnosis, situation assessment, decision support, on the basis of analysis and research, building contains a variety of human-computer interaction in the form of unmanned ground vehicles, the intelligent decision support system by determining hierarchy of human-computer interaction, so as to achieve optimal human- robot collaborative, lay a certain foundation for the research of unmanned ground vehicle autonomy.
\end{abstract}

Keywords: Unmanned Ground Vehicle, Human—Robot Coordination, Variable Autonomy, Fault Detection and Diagnosis, Intelligent Decision Support System

\section{基于人机协同的无人地面车辆智能决策支持系统构建与优化}

\author{
李静 ${ }^{1}$, 唐振民 ${ }^{2}$, 谭业发 ${ }^{1}$, 石朝侠 $^{2}$, 刘家银 $^{2}$
}

${ }^{1}$ 野战工程学院, 解放军理工大学, 江苏南京, 中国

${ }^{2}$ 计算机科学与工程学院, 南京理工大学, 江苏南京, 中国

\section{邮箱}

lijingwry@126. com（李静）, tang.zm@163.com（唐振民）, tanyefa7651@162.com（谭业发）, stonexia@soho.com（石朝侠）, smiton@foxmail.com (刘家银)

\begin{abstract}
摘要: 无人系统的自主性是一种拥有感知、观察、分析、交流、计划、制定决策和行动的能力，并且完成人类通过人 机交互布置给它的任务。地面无人系统在学术研究领域内称为移动机器人或自主陆地车, 在军队中称为无人地面车辆。 本文在对可变自主、故障诊断、态势估计、决策支持分析和研究的基础上，构建包含多种人机交互形式的无人地面车 辆智能决策支持系统, 通过确定人机交互的等级层次, 以期实现最优的人机协同, 为无人地面车辆自主性研究打下一 定基础。
\end{abstract}

关键词: 无人地面车辆, 人机协同, 可变自主, 故障检测和诊断, 智能决策支持系统 


\section{1. 引言}

地面无人系统在学术研究领域内称为移动机器人或 自主陆地车, 在军队中称为无人地面车辆 (Unmanned Ground Vehicle, UGV), 是一种能够在各种地面环境, 无 须人工干预, 连续自主运动完成任务的轮式、履带式或混 合式车辆 $[1]$ 。自主性是无人系统的一种属性, 是一种拥 有感知、观察、分析、交流、计划、制定决策和行动的能 力, 并且完成人类通过人机交互布置给它的任务 [1]。自主 性可以根据任务复杂性、环境复杂度和为了完成任务进行 的人机交互等因素来区分等级 [2]。因此, 无人系统自主 性等级与系统需要的交互信息量之间成反比关系, 即人机 交互程度越高, 系统自主性等级越低, 人机交互是人机协 同的方式。本文构建包含多种人机交互形式的无人地面车 辆智能决策支持系统, 通过确定人机交互的等级层次, 实 现最优化人机协同, 为无人地面车辆自主性研究奠定一定 基础。

\section{2. 人机协同}

在无人系统比赛或是性能演示中, 通常都是以最大程 度的展示其自主性为目的, 但在实际任务执行中, 尤其是 在战场环境中, 更多的是以安全完成任务为目的。以目前 的技术水平, 制造出与人一样具有智能思维的无人系统还 不现实, 更多时候是人与系统协同完成任务。

\section{1. 人机交互}

人机交互 (HRI) 是一个包括机器人技术、人工智能、 认知科学和人-计算机接口 (HCI) 在内的交叉学科领域, 是多个智能体在一个系统内一起工作的大体系 [3]。研究 人机交互目的就是为了解决如何使人机系统相互协同, 更 好的完成任务。只有建立有效的、灵活的、新型的人机关 系, 最终使建立的系统能够将智能计算机系统与人之间形 成一种类似于人与人之间的、交流无障碍的同事关系，各 自执行自己最擅长的任务, 形成更高智能的合作产物 [4]。

\section{2. 人、机特征}

人与无人系统都属于智能体, 具有不同的能力, 按照 人机的特征分配任务是首要解决的问题, 通过人机交互达 到人机协同是研究目的。人、机在不同方面各有特点, 人 善于定性推理、处理不明确的感知信息和突发意外状况, 具有较强的学习能力、随机应变能力, 适应性较强; 系统 能够存储大量信息, 善于做重复工作、逻辑推理、定量计 算等, 能在恶劣和危险环境中工作。只有人机各尽其用, 互相监督, 才能达到整个人机系统的高智能性。

\section{3. 不同自主性等级的人车协同}

无人地面车辆不需要驾驶员, 但仍需要操作员进行不 同程度、不同阶段、不同方面的监督控制。目前已成型的 无人地面车辆主要以 “遥控车辆” 、“遥操作” 和 “半自
主式”为主, 高自主性的无人系统也仅限于在特定环境中。 自主性等级不同, 人机协同的方式也不同。

\subsection{1. 遥控车辆}

遥控车辆一般在操作员视野范围内工作, 完全由人来 操控, 不具备接收、分析外界信息的能力, 不能进行环境 辨别, 所有的态势感知过程、行为决策都由操作员来完成, 其自主性与人的智能性和车辆自身的性能指标有关。

\subsection{2. 遥操作车辆}

遥操作不等同于遥控, 而是在无人地面车辆自主行驶 的过程中, 增加了人在回路的任务决策、环境感知与规划、 控制等功能, 具备人与车的实时通信交互, 属于提升无人 地面车辆控制能力的一种人机交互方式 [5]。

遥操作车辆具备部分自主, 对感知的数据信息具有响 应, 人对车辆只起监督指导的作用, 通过接口控制车辆的 行为, 达到在远处工作的无人地面车辆高自主性, 其自主 性取决于人和车辆智能的有机集合 $[6]$ 。

\subsection{3. 半自主式车辆}

半自主式车辆通常又被称为 “追随者”, 通过跟踪“向 导” 标定的踪迹 (通常称为 “面包屑” ) 来判定自己的周 围环境, 按已规划好的最佳路线来完成任务, 具备简单地 形推断能力, 出现突发状况请求操作员决策 [3], 其自主 性体现在系统与人进行动态合作, 任务重分配、决策重规 划的过程中。

\subsection{4. 全自主车辆}

全自主车辆的操作完全由系统操控, 人只给出任务目 标, 系统将其转化为任务, 形成规划决策, 并逐层分解至 车辆动作。人在系统运行过程中不干预, 只在系统运行过 程中进行监督, 其自主性就是无人地面车辆的自主性。

\section{3. 人机交互模式}

在由人和无人系统组成的复杂智能系统中, 人机之间 存在感知、决策规划和执行三个层次的信息融合, 结合无 人地面车辆执行任务的特点和面对的工作环境, 以及人的 监督控制确定人机交互模式。

\section{1. 可变自主}

可变自主性模式是由Alberto Valero和Massimo Mecello 提出的一种人机合作控制模式 (human一robot interaction, HRI)。

\section{1. 1 . 可变自主性概念}

可变自主性系统是由人与系统 (单个或多个智能系统) 组成, 通过高效地人机交互, 充分利用各组成个体的优势, 最好地配置系统资源, 以适应当前形势完成任务, 达到整 个系统性能最优化 [7]。无人系统自主性变化体现在必要 时将决策控制权转交给更高智能的Agent, 同时调整自身 
自主性等级, 实现在系统运行中人、机功能的动态调整, 在任务不同阶段体现不同的自主程度 [8-9]。

因此，单一的任务分配标准与方法，或是执行任务过 程中同一不变的自主性等级策略不能完全满足复杂人机 智能系统的实时需要。

\subsection{2. 可变自主性实现}

自主等级的调整改变的是人机之间的任务分配和决 策权, 属于一种动态分配。在设计支持可变自主的无人系 统时, 按人、机的特征分配最适合的任务, 通过人机接口 在最需要的时候让操作员参与进来, 而不是在单调、重复、 常规和系统能够自主完成的操作任务中参与进来 [10-11]。

在执行任务开始阶段, 系统以自身最高自主性能力开 始工作; 过程中通过感知无人地面车辆周围工作环境、自 身行驶状态和任务信息, 推断外部状况的改变对其自主性 各方面能力产生的影响, 一般分为能判断、难判断和不能 识别; 通过态势推理、威胁评估, 将能够处理的态势由系 统自主处理, 不能处理的将决策规划权转移给其他智能体 (人或更高智能的系统) ; 通过人机交互方式向操作者求 助或询问降低自主性等级, 等待处理; 当所处环境重新回 到适合系统自主完成任务的状况时, 由操作者或系统自身 来提高自主性等级, 实现由系统完成自主控制。

\section{1.3. 无人地面车辆自主性调整方式}

从无人地面车辆具备的自主性能力分析来看, 其自主 性调整有以下方式: 调节系统中不同子系统 (感知、规划、 运动控制、行为和学习能力）的自主性等级; 调节不同子 系统在完成不同任务阶段时的自主性等级 (各个任务阶段 所处的工作环境需要的自主性能力不同); 调整系统整体 自主性等级。一般由系统自行调整完成、经操作员授权后 完成或直接由操作员完成, 其中系统自身对自主性的调整 也是其自主性能力的一种体现。
具备可变自主能力的无人系统灵活性更高, 工作范围 更广，任务完成效率也大幅提升。

\section{2. 态势估计}

可变自主的基础是对当前态势的感知、分析和理解, 对未来态势的预测。态势的概念源于军事领域, 用来说明 一个较大范围的、内部结构比较复杂、受多因素影响的被 研究对象的状态综合表现 [12]。Endsley将态势估计定义 为, 对一定时空范围内环境中诸元素状态的感知, 对它们 含义的理解以及对它们未来状态的预测, 是信息的高级融 合，属于决策级方面的融合 [13]。

在无人地面车辆完成任务的过程中, 面对复杂多变的 地面环境和战场环境, 需要对态势进行完全把握才能很好 的完成任务。因此, 构建一个实际的态势评估系统, 需要 有关环境因素的数据和比对图例、作战条令条例知识、专 家经验、各种武器装备信息、以及情感、宗教等各种人文 环境因素作为背景, 必须进行大量的积累和实验。

\section{3. 智能故障诊断}

系统对出现故障的判断是其自主性的一种体现, 也是 系统进行自主性调整的一个因素。

\section{3.1. 故障概念}

故障是指装备在规定条件下不能完成其规定功能的 一种状态, 这种状态往往是由不正确的技术条件、运算逻 辑错误、零部件损坏、环境变化、错误操作等引起的 [14]。 故障诊断是对装备运行状态和异常情况做出判断, 即在故 障尚未发生前, 对装备的运行状态进行估计, 在发生故障 后, 对故障的原因、部位、类型、程度等做出判断, 并制 定维修决策, 实施必要的维修行为 [15]。

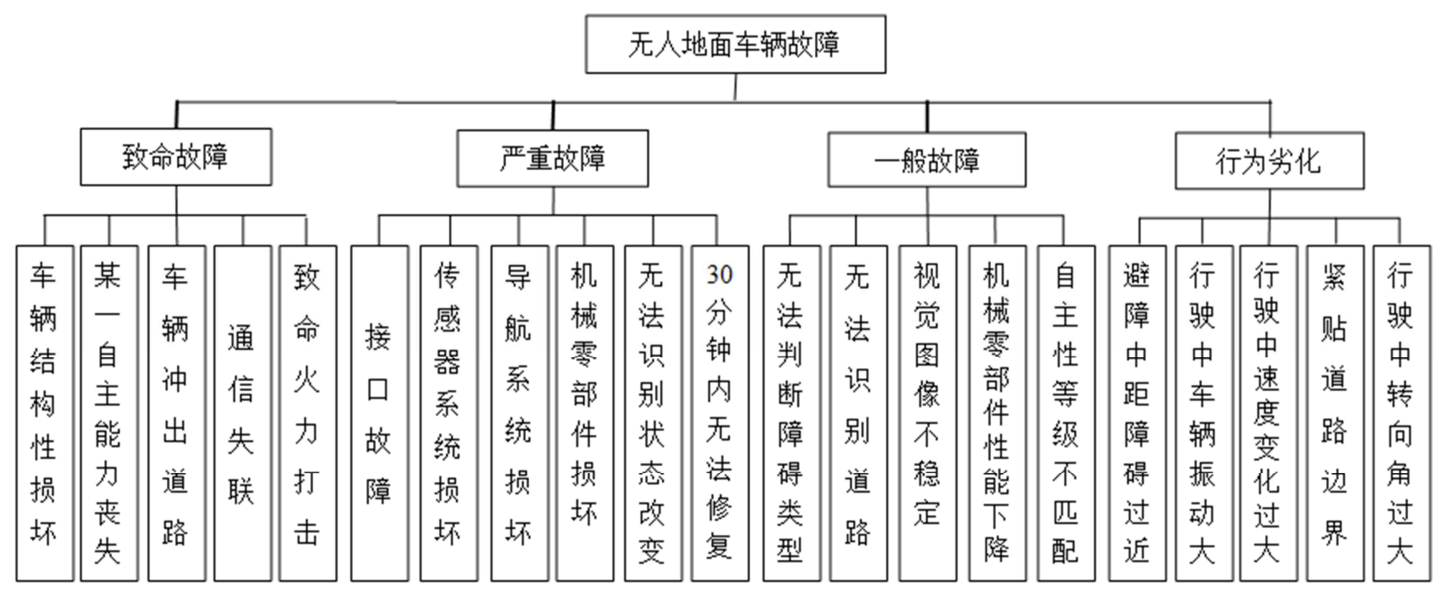

图1 无人地面车辆故障类型。

\section{3.2. 无人地面车辆故障分类}

故障分类方法较多, 根据故障发生的原因分为人为故 障和自然故障; 根据故障的危害程度、修复难度等分为致 命故障、严重故障、一般故障和轻微故障; 根据故障发生 的性质分为硬故障和软故障; 根据故障发生的位置分为对
象故障、仪表故障和系统故障 [14]。本文将无人地面车辆 的故障进行分类, 其中将因环境变化、目标改变、态势改 变等引起的不能识别、判断、处理和决策的突发事件、状 态改变, 以及一些在行驶过程中的行为劣化等都看作是故 障的表现形式, 具体如图1所示。 


\section{3. 3. 故障检测与诊断流程}

车辆的行驶轨迹是车辆行为优劣的一种反映。因此, 本文认为对轨迹的监测可以用来推断无人地面车辆故障 潜在发生的可能性，继而进行判断和决策。故障检测和诊 断都离不开人机交互手段, 是人机交互程度的一种体现, 具体流程如图2所示。

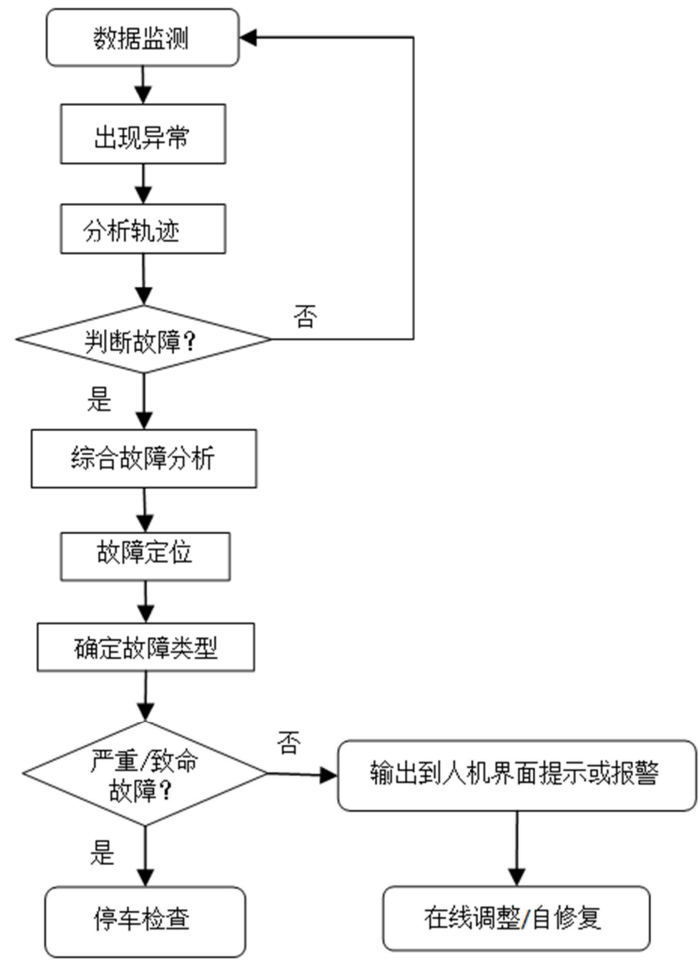

图2 无人地面车辆故障检测与诊断流程。

\section{4. 决策支持}

决策支持系统 (Decision Support System, DSS) 的概 念最早由Gorry和Scott在1971年提出的。决策支持系统不 是代替决策者进行决策, 而是为决策者提供决策所需的数 据、信息和背景资料, 帮助明确决策目标和进行问题识别, 建立或修改决策模型, 提供各种可能方案, 并对各种方案 进行评价和优选, 通过人机对话进行分析、比较和判断, 为正确决策提供必要的支持 [16-18]。

DSS 是以数据和常规数值计算方法来辅助决策, 而无 人地面车辆在现实工作环境遇到的大都是动态的、不可预 测的、非结构化的问题, 难以用数学模型来表示, 需要大 量专业知识和经验。因此, 智能决策支持系统更加适合无 人地面车辆系统, 引入专家系统, 同时在人机交互系统中 设置自然语言处理系统, 提高操作的通用性和简易性。

\section{4. 无人地面车辆智能决策支持系统体系结构}

本文建立的无人地面车辆智能决策支持系统是一种 相对理想化的系统运行状态, 是一种能够为系统提供可变 自主、态势评估和故障检测与诊断的智能决策支持系统。

\section{1 . 系统结构}

无人地面车辆在执行任务的过程中, 受大量随机因素 的影响, 面对的环境、态势和目标等方面的信息实时变化, 需要解决非结构化、多目标的决策问题。因此, 本文在对 可变自主、故障诊断、DSS、IDSS分析的基础上，根据无 人地面车辆系统结构、工作环境和执行任务的特点构建无 人地面车辆智能决策支持系统, 具体如图3所示。

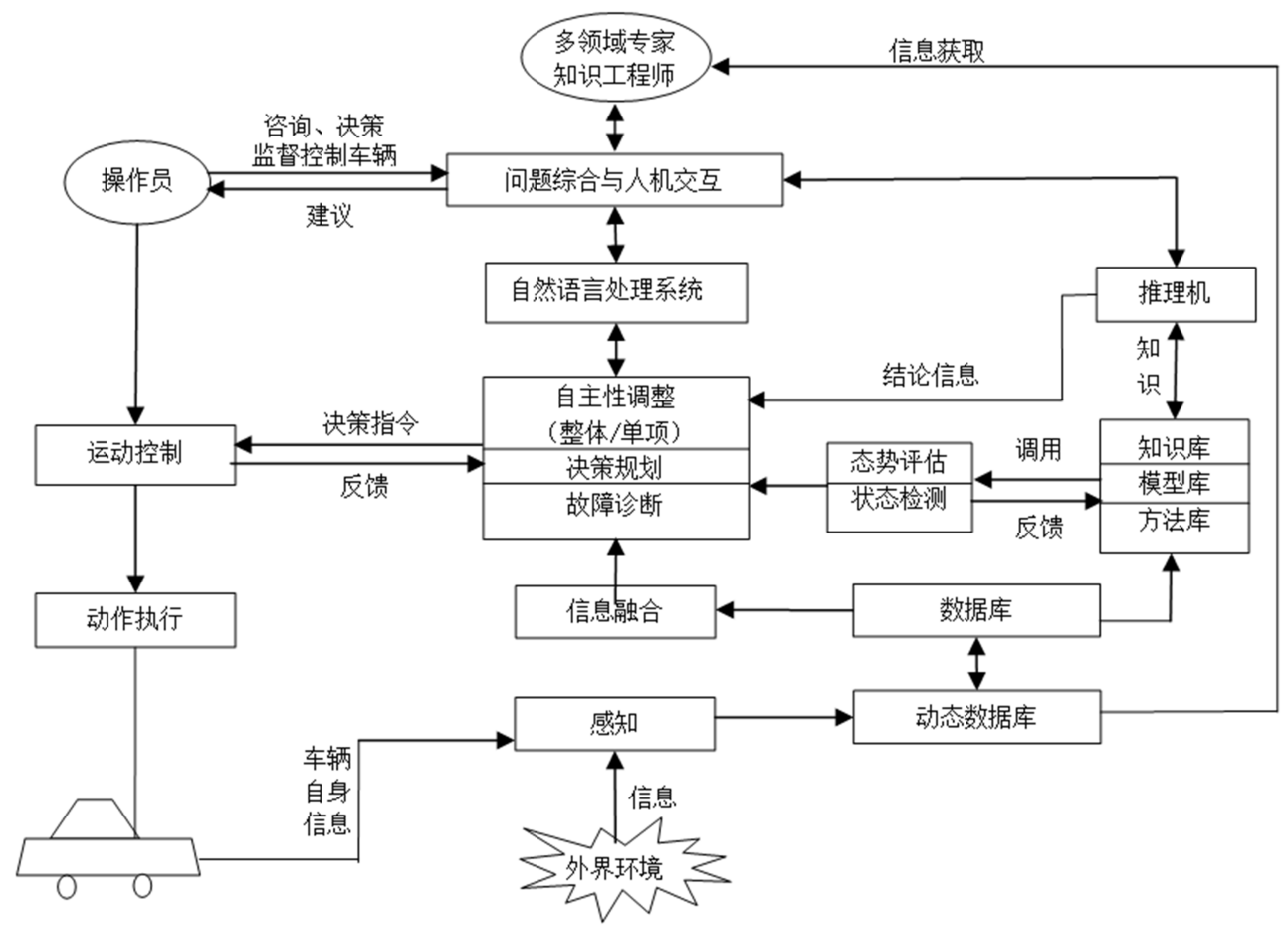

图3 无人地面车辆智能决策系统体系结构。 


\section{2. 系统组成要素}

无人地面车辆智能决策支持系统是在DSS三库结构 的基础上构建的。其中, 数据库包括各种环境组成要素 的数据信息和图像信息 (交通标识、路面材质、路侧环 境、各种障碍、作战标识、特殊障碍等), 以及无人地 面车辆在自主行驶过程中通过传感器实时感知的、经融 合的动态数据信息; 模型库存有自主性评价模型、各种 环境模型、行驶轨迹评测模型等; 方法库除了基本算法 外, 还包括信息融合方法、态势评估方法、特征提取方 法、图像处理方法、道路识别方法、评价方法、以及故 障诊断、预测和处理方法等; 知识库包括自然语言处理 所需的语言文法库和专家提供的知识（战术知识、社情 知识库等）。

所有库中的信息都可以随着研究的进展不断进行改 进和完善。

\section{3. 系统实现}

人机交互过程中设置的自然语言处理系统，使人机交 互更加简洁、方便、快捷，在战时环境中能够适应不同技 术水平的操作员; 可变自主模块中的态势评估能够根据当 前外部环境信息和任务信息，推断出外界条件的改变对无 人地面车辆的感知、运动和规划产生的影响与无人地面车 辆完成任务的能力所产生的冲突, 或是根据对未来态势的 预测调整整体或单项能力自主性等级与之匹配; 故障诊断 模块在对车辆的行驶状态 (轨迹) 进行监测的基础上, 实 时对故障的发生进行判断和预测, 得到对故障的分类、定 位和处理决策; 若行驶过程中没有突发状况出现, 车辆就 沿着规划轨迹自主行驶。

\section{5. 人机交互程度分析}

人与无人系统的数据传递、信息交流、界面操作、输 出输入指令、决策规划等都需要通过人机交互接口来完成, 人与无人系统的交互程度也从人机接口上反映出来。因此, 根据以上对无人地面车辆智能决策支持系统的构建和分 析, 本文将人机交互程度从低至高分为操作级、交流级、 调整级、决策级和控制级五个等级，同时也反映人机协同 能力的高低, 如表1所示。

\section{表 1 人机交互程度等级。}

\begin{tabular}{|c|c|c|}
\hline 等级 & 类型 & 描述 \\
\hline 1 & 操作级 & 日常界面操作、显示、动态任务受领等 \\
\hline 2 & 交流级 & $\begin{array}{l}\text { 无人系统对突发状况、决策规划、无法判断的情 } \\
\text { 况等进行判断后需人确认, 或是人经判断后给系 } \\
\text { 统提供信息输入, 以问答的方式进行交流 }\end{array}$ \\
\hline 3 & 调整级 & $\begin{array}{l}\text { 自主性的调整、在线故障维护、人对系统决策修 } \\
\text { 正等 }\end{array}$ \\
\hline 4 & 决策级 & $\begin{array}{l}\text { 由人对无人系统的运动进行规划、降低自主等级 } \\
\text { 实行遥操作 }\end{array}$ \\
\hline 5 & 控制级 & 无人系统失去了自主能力, 完全由人来操作 \\
\hline
\end{tabular}

\section{6. 结束语}

本文建立了一种理想状态的无人地面车辆智能决策 支持系统，以期通过人机交互实现可变自主、态势估计、 故障诊断、决策支持等, 最终达到以人机协同最优化的方 式高效率完成任务。并根据无人地面车辆任务特点对系统 中的各个组成部分进行分析, 确定人机交互的程度和协同 能力, 作为系统自主性研究的补充和参考。

\section{致谢}

本文为面上项目《基于场景-拓扑的多机器人协作环 境搜索研究》(61371040) 的阶段性成果之一。

\section{参考文献}

[1] Hui-Min Huang, Elena Messina, James Albus. Toward a Generic Model for Autonomy Levels for Unmanned Systems (ALFUS) [C]. Proceedings of the Performance Metrics for Intelligent Systems Workshop, Gaithersburg, Maryland, 2003.

[2] 高劲松, 李慧, 陈哨东. 美国国防部无人系统路线图中自主 性的技术演进 [J]. 国际航空. 2012, 5:36-38。

[3] 付梦印, 王美玲译. 军用无人地面车辆技术的发展 [M]. 北京: 国防工业出版社, 2009。

[4] 蒋新松. 水下机器人 [M]. 沈阳: 辽宁科学技术出版社. 2000。

[5] 李波. 人在回路的无人驾驶车辆启发式全局路径规划算法 研究 [D]. 北京: 北京理工大学, 2013。

[6] 高胜, 赵杰. 基于人机合作的遥操作机器人系统控制模型 [J]. 哈尔滨工业大学学报, 2006, 38(3) :447-451。

[7] Freed M, Bonasso P, Ingham M, et al. Trusted Autonomy for Spaceflight Systems [J]. Aiaa Journal, 2005.

[8］张国忠, 彭辉, 牛轶峰, 等. 多无人机监督控制系统的结构 分析与设计 $[\mathrm{J}]$. 系统仿真学报, 2010, 22 (A01) : 138-141。

[9] Crandall J W, Goodrich M A. Experiments in adjustable autonomy $[\mathrm{C}]$. Systems, Man, and Cybernetics, IEEE International Conference on. IEEE, 2001: 1624-1629.

[10] Endsley, M R, Kaber D B. Level of automation effects on performance [J]. Situation Awareness and Workload in a Dynamic Control Task Ergonomics, 1999, 42 (3) : 462-492.

[11] Endsleymr. Toward a theory of situation awareness in dynamic systems [J]. Human Factors, 1995, 37(1): $32-64$. 
[12] 柴慧敏. 态势估计中的关键技术研究 [D]. 西安电子科技大 学, 2009。

[13] 李伟生. 信息融合系统中态势估计技术研究 [D]. 西安电子 科技大学, 2004。

[14] 张金玉, 张炜. 装备智能故障诊断与预测 [M]. 北京: 国防工 业出版社，2012。

[15] 段琢华, 蔡自兴, 于金霞. 未知环境中移动机器人故障诊断 与容错控制技术综述 [J]. 机器人, 2005, 27 (04) : 373-379。
[16] 梁振丽, 贾晓丰. 决策支持系统理论与实践 $[M]$. 北京: 清华 大学出版社, 2014。

[17] 陈文伟. 决策支持系统及其开发 [M]. 北京: 清华大学出版社, 2014。

[18] 王媛湲, 刘海峰, 张超, 等. 信息融合中的辅助决策技术应 用概述 [C], 第三届中国指挥控制大会论文集(下册),2015。 